with an 18-gauge needle be added to your first aid kit to be used with the filtered water from your unit.

Stirling B. Smith, DO

Christopher S. Kang, MD

Tacoma, WA, USA

William Kuykendall

Seattle, WA, USA

\section{Visual Analog Scales for Assessment of Severe Acute Mountain Sickness on Aconcagua}

Background.-The Lake Louise AMS Self-report Score (LLSelf) is a commonly used, validated method of assessing acute mountain sickness (AMS).

Objectives.-Compare a visual analog scale (VAS) method of quantifying AMS with the LLSelf among trekkers to $6962 \mathrm{~m}$ for both overall symptoms and the 5 individual constituent categories of the LLSelf.

Methods.-Setting: Plaza de Mulas base camp (4365 m), Aconcagua Provincial Park, Argentina. Inclusion criteria: volunteer trekkers to Aconcagua (6962 m) in January 2009 who provided written informed consent. Exclusion criteria: pregnancy, age $<18$ years. Study protocol: Subjects were prospectively enrolled while ascending, instructed to complete a questionnaire when symptoms were maximal, and return it upon descent. The questionnaire consisted of maximum altitude reached, the LLSelf, and standard 100-mm VAS for each LLSelf component, as well as overall AMS. Statistics: means with $95 \%$ confidence interval (CI), interrater reliability with Cohen's $\kappa$, and correlation of VAS with LLSelf with Spearman's rank correlation coefficient $(r)$ were computed using Stata (Stata Corp, College Station, TX).

Results.-A total of 45 subjects completed both VAS and LLSelf. Twenty-two (49\%) climbers reached the summit (6962 $\mathrm{m})$. Mean maximum altitude reached was $6467 \mathrm{~m}$. Mean altitude of maximal symptoms was $5859 \mathrm{~m}$. Mean LLSelf was 5.1 (95\% CI, 4.3-5.9). Interrater reliability for measurement of VAS was high $(\kappa=0.9998)$. All individual VAS categories were highly correlated with their respective LLSelf symptoms: headache $r=0.85$; gastrointestinal symptoms $r=0.57$; fatigue $r=0.79$; lightheadedness $r=0.72$; and difficulty sleeping $r=$ 0.75 . Overall VAS was also highly correlated with total LLSelf: $r=0.71$.

Conclusions.- In this study of VAS and LLSelf at extreme altitude on Aconcagua (6962 m), individual VAS and overall VAS were highly correlated with both individual constituent categories of LLSelf and overall LLSelf. Further studies comparing VAS, LLSelf, and physician assessment of AMS are needed to determine optimal measurement of AMS, especially on expeditions where multiple languages are encountered.

Jon D. Van Roo, MD

Matthew P. Lazio, MD

Sanjeev Malik, MD

D. Mark Courtney, MD Chicago, IL, USA
Safety and Efficacy of Attempts to Reduce Shoulder Dislocations by Nonmedical Personnel in the Wilderness Setting

Shoulder dislocations are common in wilderness sports. Early reduction of the dislocated shoulder significantly relieves the patient's pain and increases the likelihood of a safe evacuation from the wilderness environment. Our hypothesis is that reduction attempts on scene by nonmedical personnel are safe, effective, and can significantly reduce the time to reduction.

The objectives of this study were to define the success rate and the complication rate for shoulder reduction attempts by nonmedical personnel in the wilderness setting, and to compare the average time to reduction for those done on scene versus those that waited for reduction at a medical facility.

In this prospective, cross-sectional study we administered surveys to users of wilderness sports internet forums between October 2008 and April 2009. These surveys asked respondents to describe previous wilderness sports injuries they experienced. Descriptive statistics were calculated using SPSS software, and the Mann-Whitney $U$ test was used to compare average reduction times, with an alpha of .05 selected as the significance threshold.

Overall, there were 112 responses with 58 describing shoulder dislocations that were reduced either on scene or at a medical facility. Reduction on scene, in the absence of a medically trained person, was attempted in 39 of these 58 cases with a success rate of $71.8 \%$ (28/39). The median time to reduction on scene was 10 minutes, compared to 180 minutes from the time of injury for those that waited for reduction at a medical facility $(P<.001)$. Other than pain during the reduction, there were no reports of serious complications associated with the reduction attempts. These seminal data suggest that reduction of dislocations in the wilderness setting by nonmedical personnel may be safe and effective, and can significantly decrease the time to reduction. These findings may help guide future teachings of participants in high-risk wilderness sports.

Jack Ditty, MD

Dugald Chisholm, MD

Steve Davis, MPA, MSW, LGSW

Mary Estelle-Schmidt

Morgantown, WV, USA

\section{Chronic Mountain Sickness in the Peruvian Andes}

First described by Carlos M. Monge in 1925, chronic mountain sickness (CMS), also known as Monge's disease, is the inability of usual acclimation to life at high altitudes in indigenous or long-term high altitude dwellers above $3000 \mathrm{~m}$.

Diagnostic criteria for CMS still remains controversial; however, patients will exhibit a form of erythrocytosis and a myriad 